\title{
Ground State Incongruence in 2D Spin Glasses Revisited
}

\author{
Martin Loebl* \\ Department of Applied Mathematics \\ and \\ Institute for Theoretical Computer Science (ITI) \\ Charles University \\ Malostranske n. 25 \\ 11800 Praha 1 \\ Czech Republic \\ loebl@kam.mff.cuni.cz
}

Submitted: Jan 27, 2004; Accepted: Jun 11, 2004; Published: Jun 16, 2004

MR Subject Classifications: 82D30,05C90, 05C80

\begin{abstract}
A construction supporting a conjecture that different ground state pairs exist in the 2-dimensional Edwards-Anderson Ising spin glass is presented.
\end{abstract}

\section{Introduction}

A fundamental and extensively studied problem on the way towards understanding the effects of disorder and frustration is to determine the multiplicity of infinite volume groundstates in finite-dimensional realistic models. One conjecture, in analogy with the meanfield Sherrington-Kirkpatrick model, is that finite-dimensional short-ranged systems with frustration have infinitely many groundstate pairs ([2], [3]). A different conjecture based on droplet-scaling theories predicts that only one groundstate pair exists ([4], [5], [6]).

The simplest system used to study these questions is the Edwards-Anderson Ising model ([1]) in dimension two. Here the hypothesis that only one groundstate pair exists has received support from seminal analytic work of Newman and Stein ([8], [7]). The purpose of this paper is to present a combinatorial construction supporting the competing

\footnotetext{
${ }^{*}$ Gratefully acknowledges the support of ICM-P01-05, Project LN00A056 and GAUK 158. This work was completed while visiting the DIM, U. Chile.
} 
hypothesis. In particular very intuitive Conjectures 4,5 are formulated whose validity implies that incongruent (finitely incongruent respectively) groundstate pairs exist. These conjectures concern only finite sublattices of the square lattice and hence they may be studied by many tools including computer simulations.

The Edwards-Anderson Ising model on a graph $G=(V, E)$ is defined by coupling constants $J_{i j}$ assigned to each edge $\{i, j\}$ of $G$. We will assume that $J_{i j}$ 's are independently chosen from a mean zero Gaussian distribution. A physical state of the system is given by a spin assignment $\sigma: V \rightarrow\{ \pm 1\}$ which has the corresponding energy

$$
E(\sigma)=-\sum_{\{i, j\} \in E} J_{i j} \sigma_{i} \sigma_{j}
$$

A state is groundstate if its energy cannot be lowered by changing an arbitrary finite set of spins. Groundstates exist for the square lattice $\mathcal{S}$ and arbitrary coupling constants $J_{i j}$ assigned to its edges by a compactness argument. Note that if we reverse all spins in a groundstate we again get a groundstate. Let us call these pairs groundstate pairs, or GSPs. Edge $i j$ is satisfied by spin assignment $\sigma$ if $J_{i j} \sigma_{i} \sigma_{j}>0$. Two GSPs are called incongruent if the set of edges satisfied by exactly one of them has a positive density. Note that the connectivity components of such a set in the dual lattice are sometimes called 'domain walls'.

In 1D there is no frustration and only a single GSP exists. In other dimensions the main incongruency problem may be formulated as follows:

Conjecture 1 Let the coupling constants $J_{i j}$ in the square lattice be chosen at random. Then with probability strictly bigger than zero there are incongruent GSP's.

In their strategy to prove that incongruent GSP's do not exist in the 2-dimensional square lattice, Newman and Stein ([8], [7]) approach the main problem by means of metastates. A metastate has been introduced as a translation invariant measure constructed as follows: for each finite sublattice $S_{L}$ of the square lattice $\Sigma$ with periodic boundary conditions consider the joint distribution of coupling constants and GSPs in $S_{L}$. When $L$ goes to infinity, by compactness, there is a subsequence of $L$ 's so that the joint distributions converge to translation-invariant (since periodic boundary conditions are imposed) joint measure. The metastate induces a translation invariant measure on the sets of edges satisfied by exactly one of two GSPs. In this setting Newman and Stein formulate their conjecture as follows:

Conjecture 2 Two randomly chosen GSPs (from the same metastate) are not incongruent.

Note an important fact (see Lemma 2 of [7]) which follows from the translation invariance: if two randomly chosen GSPs from a metastate are distinct, then with probability one they are incongruent. Newman and Stein give support to their conjecture in [7]. In particular they show that if two GSPs chosen at random from a metastate are distinct then there is exactly one domain wall between them and it is a both-ways-infinite 
path. Newman and Stein consider this situation unlikely and express belief that all the metastates are the same.

In this paper we consider the finite sublattices with different than periodic boundary conditions: we fix the spins along the boundary of the finite sublattice so that maximum number of the edges of the boundary are satisfied. Note an important fact: a state of minimum energy with these boundary conditions need not be a groundstate. We will call it a $c$-groundstate. Apart of the traditional incongruency we also consider a weaker notion: we say that two states are finitely incongruent if at least one of the domain walls between them contains an infinite both ways unbounded path. This is certainly a weaker notion of incongruency than the one of Newman and Stein but, also in view of their results described above, the existence of finitely incongruent states would support a conjecture that incongruent states exist. In our setting a weaker incongruency conjecture than Conjecture 1 may be formulated as follows:

Conjecture 3 Let coupling constants $J_{i j}$ in the square lattice $\Sigma$ be chosen at random. Then with probability strictly bigger than zero there is a nested sequence $S_{i}, i=1,2, \ldots$ of finite sublattices converging to the square lattice $\Sigma$ so that if $e_{j}$ is a c-groundstate in $S_{2 j}$ and $o_{j}$ is a c-groundstate in $S_{2 j-1}$ then both sequences $\left(o_{j}\right),\left(e_{j}\right)$ converge and their limits o, e are finitely incongruent states of $\Sigma$.

Note that $o, e$ are groundstates of $\Sigma$ since the boundary conditions 'disappear to the infinity'. Also note that the boundary conditions mean that all or all but one boundary edges are to be satisfied depending on the parity of the number of edges with negative coupling constants.

We start by considering the strip lattice $\mathcal{C}_{k}$ : the vertical coordinates of its vertices are arbitrary integers, and its horisontal coordinates run through integers from $-k$ to $k$. As an introduction to our method we show in section 2 that the strip lattice satisfies Conjecture 3. In section 3 we formulate two conjectures which imply Conjectures 3 and 1. The important feature of these new conjectures is that they concern finite sublattices only, and hence allow direct study by discrete methods and simulations. In section 4 we prove a (rather weak) consequence of Conjecture 4. The discrete aspect of Conjectures 4, 5 is supported in the last section 5 where we show that the dual formulations of the two conjectures are statements about $T$-joins in finite square lattices; $T$-join belongs to basic discrete optimization notions and as such it is heavily studied by discrete and computational methods.

\section{The strip Lattice}

Let $C(n, k)$ be the finite induced subgraph of $\mathcal{C}_{k}$ with the vertices $(i, j):|j| \leq n$. The basic building blocks of strip and square lattices are unit squares called plaquettes. A plaquette is frustrated if it has odd number of edges (out of 4) with negative coupling constants, and happy otherwise. Observe that a plaquette is frustrated if and only if arbitrary spin assignment satisfies an odd number of its edges. We define graph $C(n, k)^{*}$ to be the graph 
obtained by deletion of the vertex corresponding to the outer face of the geometric dual of $C(n, k)$. Hence the vertices of $C(n, k)^{*}$ are all the plaquettes of $C(n, k)$ and the edges are all edges $e^{*}$ such that $e$ is an edge of $C(n, k)$ not on the boundary; edge $e^{*}$ connects two plaquettes $p, q$ such that edge $e$ lies on the boundary of $p$ and $q$. If $A$ is a subset of edges of $C(n, k)$ then let $A^{*}$ denote the set of 'dual' edges: $A^{*}=\left\{e^{*} ; e \in A\right\}$. Let $n>m$, and consider the graph $D(n, m, k)=C(n, k)-C(m-1, k)$. Note that $D(n, m, k)$ has two connectivity components, each of them consists of $n-m$ horizontal levels of plaquettes. The two components of $D(n, m, k)$ are naturally called upper and lower and denoted by $D U(n, m, k)$ and $D L(n, m, k)$.

Definition 2.1 We say that $C(n, k), C(m, k)$ is a regular pair if for each $k^{\prime} \leq k$, both $C\left(n, k^{\prime}\right)$ and $C\left(m, k^{\prime}\right)$ have an even number of boundary edges with negative coupling constants and both $D U(n, m, k)$ and $D L(n, m, k)$ have exactly one frustrated plaquette, located in the middle of the lowest (highest respectively) horizontal row.

We will use the following key observation:

Lemma 2.2 Let $C(n, k), C(m, k)$ be a regular pair. Let $c(i)$ be a c-groundstate of $C(i, k)$ and let DIS $(c(i))$ denote the set of edges dissatisfied by $c(i), i=n, m$. Then the symmetric difference $D I S(c(n))^{*} \Delta D I S(c(m))^{*}$ contains a path from a plaquette of $D U(n, m, k)$ to a plaquette of $D L(n, m, k)$.

Proof. The subgraph formed by $D I S(c(i))^{*}$ induces odd degree in each frustrated plaquette and even degree in each happy plaquette of $C(i, k)$. Moreover for $i=m, n, D I S(c(i))$ contains no edge of the boundary of $C(i, k)$. Hence $D I S(c(n))^{*} \Delta D I S(c(m))^{*}$ induces odd degree in each frustrated plaquette of $D(n, m, k)$, and even degree in arbitrary other plaquette of $C(n, k)$. This easily implies the Lemma.

Now we are ready to show that

Conjecture 3 holds for the strip lattice:

Let $S_{i}=C(i, k)$. Clearly, for almost all coupling constants assignments $J$ in the whole strip lattice there is a sequence $\left(m_{j}\right)$ so that for each $j, C\left(m_{j}, k\right), C\left(m_{j}-1, k\right)$ is a regular pair. Let $o_{j}$ be a c-groundstate in $S_{m_{j}}$ and let $e_{j}$ be a c-groundstate in $S_{m_{j}-1}$. Lemma 2.2 implies that for each $j, D I S\left(o_{j}\right)^{*} \Delta D I S\left(e_{j}\right)^{*}$ contains a path $P_{j}$ of length at least $2 j+1$. Now it is easy to see that

Claim 1. There is a subsequence $P_{n_{j}}$ that converges to both ways infinite path $P$.

By compactness there is a subsequence $\left(p_{j}\right)$ of $\left(n_{j}\right)$ so that both sequences $\left(e_{p_{j}}\right)$ and $\left(o_{p_{j}}\right)$ converge. Let the respective limits be $e$ and $o$. Then necessarily $P$ is a subset of

a domain wall between $e$ and $o$ and so $e, o$ are finitely incongruent. Hence Conjecture 3 holds for the strip lattice.

\section{The Finite Conjectures}

In this section we formulate Conjecture 4 and Conjecture 5. We show that Conjecture 4 implies Conjecture 3 and Conjecture 5 implies the main Conjecture 1. 
Definition 3.1 We first introduce some notation.

- We denote by $\mathbf{A}(\mathbf{n}, \mathbf{k})$ the set of all coupling constants assignments in $\Sigma$ so that there is a c-groundstate $r$ in $C(n-1, k)$ and a c-groundstate $s$ in $C(n, k)$ and a path $P$ in $D I S(r)^{*} \Delta D I S(s)^{*}$ from a frustrated plaquette of $D U(n, n-1, k)$ to a frustrated plaquette of $D L(n, n-1, k)$ which contains an edge in distance at most 100 from the origin. The distance here means the length of a shortest path in $C(n, k)$ form the origin to a plaquette which forms an end-vertex of the edge of $P$.

- A row $R$ of plaquettes of $C(n, k)$ is called isolation if the middle plaquette $M$ is the only frustrated one and for each horizontal edge e $\notin M,\left|J_{e}\right|>\sum_{e^{\prime}}\left|J_{e^{\prime}}\right|$ where the sum is over all edges $e^{\prime}$ such that $e^{\prime} \in M$ or $e^{\prime}$ is a vertical edge incident to a vertex of a plaquette of $R . C(n, k)$ is called isolated if both boundary horizontal levels of plaquettes are isolation.

- We denote by $\mathbf{R}(\mathbf{n}, \mathbf{k})$ the set of all coupling constants assignments in $\Sigma$ so that $C(n, k), C(n-1, k)$ is a regular pair and $C(n, k)$ is isolated.

- Finally we denote by $\mathbf{R}^{\prime}(\mathbf{n}, \mathbf{k})$ the set of all coupling constants assignments in $\Sigma$ which belong to $R(n, k)$ and do not belong to $R\left(n^{\prime}, k\right)$ for $k \leq n^{\prime}<n$.

In Figure 1 below path $P$ is depicted. Dotted line corresponds to $D I S(s)^{*}$, solid line corresponds to $D I S(r)^{*}$ and little squares lie in the frustrated plaquettes.

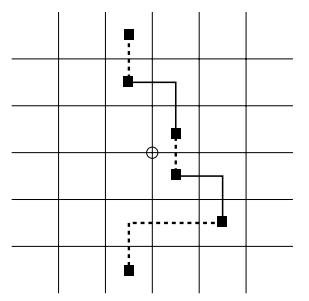

Figure 1.

We will show that the following conjecture implies Conjecture 3 .

Conjecture 4 There is positive integer constant $c$ and $\epsilon>0$ so that if $n, k>c+1$ then the probability of $A(n, k)$, in the set of the coupling constants assignments $J$ of $\Sigma$ such that $J \in A\left(n, k^{\prime}\right)$ for each $c+1<k^{\prime}<k$ and $J \in R^{\prime}(n, k)$, is at least $1-(k-c)^{-1}\left(\log (k-c)^{-1-\epsilon}\right.$.

How is it possible that for each $k^{\prime}<k, A\left(n, k^{\prime}\right)$ holds and $A(n, k)$ does not hold? There may be a block of heavy edges of width $2 k-1$ encircling the origin. The domain walls in $C\left(n, k^{\prime}\right), k^{\prime}<k$, pass through it since they cannot escape elsewhere however the domain wall in $C(n, k)$ avoids it. If this is essentially the only possibility, Conjecture 4 should be true.

If Conjecture 3 holds then it is natural to expect that for large $k$, a neighbourhood of the origin behaves in a similar way as the origin itself. This leads to a bolder Conjecture 5 which we will show implies the main Conjecture 1. 
Definition 3.2 We denote by $\mathbf{B A}(\mathbf{n}, \mathbf{k})$ the set of all coupling constants assignments in $\Sigma$ so that there is a c-groundstate $r$ in $C(n-1, k)$ and a c-groundstate $s$ in $C(n, k)$ and a path $P$ in $D I S(r)^{*} \Delta D I S(s)^{*}$ from a frustrated plaquette of $D U(n, n-1, k)$ to a frustrated plaquette of $D L(n, n-1, k)$ so that $P$ contains an edge in distance at most 100 from the origin $A N D$ has density at least 1/100 in the square centered at the origin with the side-length $100 k^{1 / 100}$.

Conjecture 5 Conjecture 4 holds also with $A\left(n, k^{\prime}\right)$ replaced by $B A\left(n, k^{\prime}\right), k^{\prime} \leq k$.

Theorem 1 Conjecture 4 implies Conjecture 3.

\section{Proof.}

We prove this theorem in a series of observations.

Observation 1: Let $C(n, k) \in R(n, k)$. Let $r$ be a c-groundstate in $C(n-1, k), s$ be a c-groundstate in $C(n, k)$ and let $P$ be a path in $D I S(r)^{*} \Delta D I S(s)^{*}$ from the frustrated plaquette of $D U(n, n-1, k)$ to the frustrated plaquette of $D L(n, n-1, k)$. Moreover let $n^{\prime}>n$, let $C\left(n^{\prime}, k\right), C\left(n^{\prime}-1, k\right)$ be a regular pair and let $r^{\prime}, s^{\prime}, P^{\prime}$ be defined analogously as $r, s, P$. Then $P^{\prime}$ contains $P$.

Let $k>c+1$ and let $I_{k}$ denote the set of all coupling constants assignments in $\Sigma$ so that for each $c+1<k^{\prime} \leq k$ there is $n\left(k^{\prime}\right) \geq k^{\prime}$ so that $C\left(n\left(k^{\prime}\right), k^{\prime}\right) \in R\left(n\left(k^{\prime}\right), k^{\prime}\right) \cap A\left(n\left(k^{\prime}\right), k^{\prime}\right)$.

Observation 2: If $k>c+1$ and Conjecture 4 holds then

$$
\operatorname{Prob}\left(I_{k}\right)=\operatorname{Prob}\left(\cap_{c+1<k^{\prime} \leq k} I_{k^{\prime}}\right) \geq \prod_{j=c+2}^{k}\left(1-(j-c)^{-1}(\log (j-c))^{-1-\epsilon}\right) .
$$

Proof.

First notice that for each $k$ with probability one there is an infinite sequence $\left(n_{i}\right)$ such that $R\left(n_{i}, k\right)$ holds for each $i$. In particular if we let $Z(k)$ be the event 'There is no $n \geq k$ with $R(n, k)^{\prime}$, then $Z(k)$ has probability zero.

We proceed by induction on $k$. The case $k=c+2$ follows from the fact above and Conjecture 4. For the induction step first note that event $Z(k)$ has probability zero and so it also has probability zero conditioned on $I_{k-1}$, if we use the induction assumption. Hence for almost all elements of $I_{k-1}$ there is smallest $n \geq k$ such that $R(n, k)$ holds. Next note that Observation 1 implies that the set of instances satisfying $R^{\prime}(n, k)$ and $I_{k-1}$ is the same as the set of instances satisfying $R^{\prime}(n, k)$ and $A\left(n, k^{\prime}\right)$ for each $c+1<k^{\prime}<k$. Hence assuming validity of Conjecture 4 and the induction assumption we get

$$
\begin{gathered}
\operatorname{Prob}\left(I_{k}\right)=\operatorname{Prob}\left(I_{k-1}\right) \operatorname{Prob}\left(I_{k} \| I_{k-1}\right)= \\
\operatorname{Prob}\left(I_{k-1}\right) \sum_{m} \operatorname{Prob}\left(R^{\prime}(m, k) \| I_{k-1}\right) \operatorname{Prob}\left(I_{k} \| R^{\prime}(m, k), I_{k-1}\right)= \\
\operatorname{Prob}\left(I_{k-1}\right) \sum_{m} \operatorname{Prob}\left(R^{\prime}(m, k) \| I_{k-1}\right) \operatorname{Prob}\left(I_{k} \| R^{\prime}(m, k), A\left(m, k^{\prime}\right), c+1<k^{\prime}<k\right)=
\end{gathered}
$$




$$
\begin{gathered}
\operatorname{Prob}\left(I_{k-1}\right) \sum_{m} \operatorname{Prob}\left(R^{\prime}(m, k) \| I_{k-1}\right) \operatorname{Prob}\left(A(m, k) \| R^{\prime}(m, k), A\left(m, k^{\prime}\right), c+1<k^{\prime}<k\right) \geq \\
\sum_{m} \operatorname{Prob}\left(R^{\prime}(m, k) \| I_{k-1}\right) \prod_{j=c+2}^{k}\left(1-(j-c)^{-1}(\log (j-c))^{-1-\epsilon}\right)= \\
\prod_{j=c+2}^{k}\left(1-(j-c)^{-1}(\log (j-c))^{-1-\epsilon}\right) .
\end{gathered}
$$

This finishes the proof of Observation 2.

Hence assuming validity of Conjecture 4 the probability of the event 'For each $k, I_{k}$ ' is at least $\prod_{j \geq 2}\left(1-j^{-1}(\log j)^{-1-\epsilon}>0\right.$. This proves Theorem 1 in the same way as Claim 2 proves Conjecture 3 for the strip lattice.

Theorem 2 Conjecture 5 implies Conjecture 1.

Proof. We proceed similarly as in the proof of the previous theorem. Let $k>c+1$ and let $B I_{k}$ denote the set of all coupling constants assignments in $\Sigma$ so that for each $c+1<k^{\prime} \leq k$ there is $n\left(k^{\prime}\right) \geq k^{\prime}$ so that $C\left(n\left(k^{\prime}\right), k^{\prime}\right) \in R\left(n\left(k^{\prime}\right), k^{\prime}\right) \cap B A\left(n\left(k^{\prime}\right), k^{\prime}\right)$. We observe as before if $k>c+1$ and Conjecture 5 holds then

$$
\operatorname{Prob}\left(B I_{k}\right)=\operatorname{Prob}\left(\cap_{c+1<k^{\prime} \leq k} B I_{k^{\prime}}\right) \geq \prod_{j=c+2}^{k}\left(1-(j-c)^{-1}(\log (j-c))^{-1-\epsilon}\right) .
$$

Hence assuming validity of Conjecture 5 the probability of the event 'For each $k, B I_{k}$ ' is at least $\prod_{j \geq 2}\left(1-j^{-1}(\log j)^{-1-\epsilon}>0\right.$. This proves Theorem 2: in the same way as above we can grow a path in the symmetric difference, so that it goes near to origin AND gradually 'fills' the whole square grid.

\section{The Pinning Lemma}

In the rest of the paper we collect support for Conjecture 4. First we present the following Pinning Lemma.

Lemma 4.1 There is a function $g$ from positive integers to $(0,1)$ so that for each $k$ and $n>k$, if coupling constants in $C(n, k)$ are chosen at random so that $C(n, k), C(n-1, k)$ is a regular pair then the probability of $A(n, k)$ is at least $g(k)$.

We postpone the proof to the appendix. Anyway, the present proof is not satisfactory. Although $g$ may well be a constant, at present we are able to show only a very weak inverse exponential lower bound for it. Next we prove a consequence of Conjecture 3, using the Pinning Lemma. 
Theorem 3 Let $\left(S_{i}=C\left(l_{i}, p_{i}\right), l_{i}>p_{i}\right)$ be a nested sequence of finite sublattices monotonically converging to the square lattice and such that for each $k$ there are sufficiently many lattices with width $k$. Let $\mathcal{J}_{i}$ be the distribution of the coupling constants in $S_{i}$. Then for almost all $\left(J_{i}\right)_{i \geq 1}$ from $\left(\mathcal{J}_{i}\right)_{i \geq 1}$ there is a converging subsequence $\left(J_{m_{j}}\right)$ with the following property: if $e_{j}$ is a c-groundstate in $S_{m_{j}}$ and $o_{j}$ is a c-groundstate in $S_{m_{j}-1}$ (with coupling constants given by $\left.J_{m_{j}}\right)$ then both sequences $\left(o_{j}\right),\left(e_{j}\right)$ converge and their limits $o, e$ are weakly incongruent states.

\section{Proof.}

Theorem 3 follows from Claim 2 below in the same way as Conjecture 3 for the strip lattices follows from Claim 1.

Claim 2. For almost all $\left(J_{i}\right)_{i \geq 1}$ from $\left(\mathcal{J}_{i}\right)_{i \geq 1}$ there is a subsequence $\left(C\left(n_{i}, k_{i}\right)\right)$ of $\left(S_{i}\right)$ so that for each $i, C\left(n_{i}, k_{i}\right)$ and $C\left(n_{i}-1, k_{i}\right)$ with the coupling constants given by $J_{n_{i}}$ is a regular pair and there is a path $P_{i}$ in $D I S\left(o_{i}\right)^{*} \Delta D I S\left(e_{i}\right)^{*}$ from a frustrated plaquette of $D U\left(n_{i}, n_{i}-1, k\right)$ to a frustrated plaquette of $D L\left(n_{i}, n_{i}-1, k\right)$ which contains an edge in distance at most 100 from the origin. Here $o_{i}$ is a c-groundstate in $C\left(n_{i}-1, k_{i}\right)$ and $e_{i}$ is a c-groundstate in $C\left(n_{i}, k_{i}\right)$.

Let $k$ be an arbitrary positive integer. Since $p_{i}=k$ for sufficiently many $i$ 's, we know by the Pinning Lemma that the probability that one of $C\left(l_{i}, k\right)$ satisfies the properties of Claim 2 is very large. Hence the set of instances $\left(J_{i}\right)_{i \geq 1}$ from $\left(\mathcal{J}_{i}\right)_{i \geq 1}$ for which the propertiess of Claim 2 donot hold has measure zero. This finishes the proof of Claim 2 and Theorem 3.

Claim 2 is a consequence of the Pinning Lemma and the fact that in each $S_{i}$ we assign the coupling constants independently. The remaining obstacle in proving the full Conjecture 3 is that because of dependancies the Pinning Lemma cannot be used independently in each $S_{i}$. Conjecture 4 may be viewed as an attempt to make the dependencies work for us.

\section{The Dual Formulation}

It seems very natural to formulate the Pinning Lemma as a property of the dual lattice. In doing so we connect our considerations with the concept of $T$-joins extensively studied in discrete optimization. This may be particularly useful for studying Conjectures 4, 5 computationally. In fact, the Pinning Lemma is proved in its dual form in the appendix. We start by listing some simple properties of lattices $C(n, k)$.

1. $C(n, k)$ has an even number of negative coupling constants on the boundary if and only if it has an even number of frustrated plaquettes.

2. A set $R$ of edges of $C(n, k)$ not on the boundary is the set $D I S(r)$ of the dissatisfied edges of a state $r$ (not necessarily a groundstate) if and only if $R$ has an odd number of edges from each frustrated plaquette and an even number of edges from any other plaquette. 
3. A state $r$ is a c-groundstate if and only if it satisfies the boundary conditions and $\sum_{(i j) \in D I S(r)}\left|J_{i j}\right|$ is as small as possible. Hence there is a natural bijection between the c-groundstate pairs of $C(n, k)$ and the sets $A$ of edges not on the boundary and satisfying: a plaquette has an odd number of edges of $A$ if and only if it is frustrated, and $\sum_{(i j) \in A}\left|J_{i j}\right|$ is as small as possible.

This means that regarding the Pinning Lemma we need only a subset of information given by the coupling constants: we need to know the value $\left|J_{i j}\right|$ for each edge $(i j)$ not on the boundary, and we need to know which plaquette is frustrated. Each plaquette is equally likely to be frustrated or happy. If $\mathcal{J}$ is our distribution of coupling constants then we denote by $|\mathcal{J}|$ the distribution of their absolute values. We are interested only in those $C(n, k)$ that contain an even number of frustrated plaquettes. Hence instead of choosing the coupling constants from $\mathcal{J}$, we can choose them from $|\mathcal{J}|$ and choose uniformly at random an even set of plaquettes which we want to be frustrated. This means that the Pinning Lemma is about $C(n, k)^{*}$ rather than about $C(n, k) . C(n, k)^{*}$ is also a square grid, of width $2 k$ and height $2 n$. We need one more definition.

Definition 5.1 Let $G=(V, E)$ be a graph and let $T$ be a subset of an even number of vertices of $G$. We say that a set $A$ of edges of $G$ is a $T$-join if each vertex $x$ of $G$ is incident with an even number of edges of $A$ if and only if $x \notin T$.

Taking these considerations into account, note that the following Lemma 5.4 implies Pinning Lemma 4.1, and Conjectures 6, 7 are equivalent dual formulations of Conjectures 4. 5 .

We denote by $C^{\prime}(n, k)$ the graph obtained from $C(n, k)$ by attaching two more vertices $[0, n+1]$ and $[0,-n-1]$ by the corresponding two vertical edges. We say that $C(n, k)$ is dually isolated if each of its two boundary rows of vertices are dual isolation. A row $R$ of vertices is dual isolation if it has exactly one vertex $r$ of $T$, located in the middle of $R$, and the weight of each vertical edge incident to a vertex of $R-r$ is bigger than the sum of the weights of the horizontal edges in $R$ and in the two rows adjacent to $R$, and the edges adjacent to $r$.

Definition 5.2 Let $D(n, k)$ denote the following property: there is a minimum $T$-join $r$ in $C(n, k)$ and a minimum $(T \cup\{[0, n+1],[0,-n-1]\})$-join $s$ in $C^{\prime}(n, k)$ so that a path $P$ from $[0, n+1]$ to $[0,-n-1]$ in $r \Delta s$ contains an edge in distance at most 100 from the origin.

Definition 5.3 Let $B D(n, k)$ denote the following property: there is a minimum $T$-join $r$ in $C(n, k)$ and a minimum $(T \cup\{[0, n+1],[0,-n-1]\})$-join $s$ in $C^{\prime}(n, k)$ so that a path $P$ from $[0, n+1]$ to $[0,-n-1]$ in $r \Delta s$ contains an edge in distance at most 100 from the origin AND has density at least 1/100 in the square centered at the origin with the side-length $100 k^{1 / 100}$.

Conjecture 6 There is positive integer constant $c$ and $\epsilon>0$ so that if $n, k>c+1$, and the absolute values of the coupling constants in $C(n, k)$ are chosen at random from $|\mathcal{J}|$, and a subset $T$ of vertices of $C(n, k)$ be chosen uniformly at random so that: 
- for each $k^{\prime} \leq k,\left|T \cap C\left(n, k^{\prime}\right)\right|$ is even,

- if $n^{\prime} \leq n$ then $C\left(n^{\prime}, k\right)$ is not dually isolated,

- for each $c+1<k^{\prime}<k, D\left(n, k^{\prime}\right)$ holds,

then the probability of $D(n, k)$ is at least $1-(k-c)^{-1}(\log (k-c))^{-1-\epsilon}$.

Conjecture 7 Conjecture 6 holds with $D\left(n, k^{\prime}\right)$ replaced by $B D\left(n, k^{\prime}\right), k^{\prime} \leq k$.

Lemma 5.4 There is a function $g$ from positive integers to $(0,1)$ so that for each $k$ and $n>k$, if we choose absolute values of coupling constants of $C(n, k)$ at random from $|\mathcal{J}|$ and choose a subset $T$ of even number of vertices of $C(n, k)$ uniformly at random then the probability of $D(n, k)$ is at least $g(k)$.

Conclusion. In this paper we formulate Conjectures 4, 5 whose validity implies that finitely incongruent and incongruent groundstate pairs exist in the 2-dimensional Edwards-Anderson Ising spin glass. The conjectures deal with finite sublattices only and may be naturally and effectively studied by computer simulations. We gather supporting evidence, namely we prove a Pinning Lemma and as its consequence a weaker statement in Theorem 3.

\section{A Proof of the Dual Pinning Lemma 5.4}

We will consider set $\mathcal{K}$ of configurations with joint distribution $\mathcal{U}_{k}$. A configuration is a pair $(J, T)$ where $J$ consists of the coupling constants and $T$ consists of an even number of vertices of $C(n, k)$. We show that there is a function $F$ from $\mathcal{K}$ to itself such that

- $\mathcal{U}_{k}(F(\mathcal{K})) \geq c_{k}>0$, and

- Each $L \in F(\mathcal{K})$ is positive, i.e. there is a minimum $T$-join $r$ in $C(n, k)$ and a minimum $(T \cup\{[0, n+1],[0,-n-1]\})$-join $s$ in $C^{\prime}(n, k)$ such that path $P$ from $[0, n+1]$ to $[0,-n-1]$ in $r \Delta s$ contains an edge in distance at most 100 from the origin.

Fix an arbitrary positive configuration $K_{0}$. Let $K=(J, T)$ be a configuration. If there is an edge $e$ incident to a vertex $[i, j]$ with $|i| \leq 4,|j| \leq k$ and $\left|J_{e}\right| \geq 1$ then let $f(K)=K_{0}$, otherwise let $P, r, s$ be as in the statement of Lemma 5.4. If $P$ passes in distance at most 100 from the origin then let $f(K)=K$. Otherwise let $m$ be the smallest positive integer such that $P$ contains a vertical edge with the $\mathrm{x}$-coordinate of its vertices equal to $m$ or $-m$ and with the absolute value of both y-coordinates at most 4 . Note that $P$ has no vertex $[i, j]$ with $|i|<m$ and $|j|<4$. Let $Z$ be the graph induced on the vertices $[i, j] ;|i| \leq m,|j| \leq 4$. We let $K=K_{1}, P=P_{1}, r=r_{1}, s=s_{1}, Z=Z_{1}, T=T_{1}$, $m=m_{1}$, let $n_{1}$ be the number of vertical edges of $P \cap Z$ and let $p_{1}$ be the number of (all) edges of $P \cap Z$. 
Next we describe a procedure with input $I_{i}=\left(K_{i}=\left(J_{i}, T_{i}, x, y\right), P_{i}, r_{i}, s_{i}, Z_{i}, m_{i}, p_{i}\right)$ which produces $F(K)$ or $I_{i+1}$.

The Procedure. If $r_{i} \Delta s_{i}$ contains a cycle then let $F(K)=K_{0}$. Otherwise let $H_{i}$ be the segment of $P_{i} \cap Z_{i}$ defined as follows: If $P_{i}$ contains a vertical edge $e=\{[z, a],[z, b]\}$ so that $|z|=m_{i}$ and $|a|<|b|<4$ then let $H_{i}$ consist of $e$. If $P_{i}$ contains no such vertical edge but it does contain a horizontal edge $e=\{[a, z],[b, z]\}$ so that $|z|=4$ and $|a|<|b|<m_{i}$ then again let $H_{i}$ consist of $e$. Finally let there be only 'corner' edges in $P_{i} \cap Z_{i}$. Let $e$ be such vertical edge (it exists by the choice of $m_{i}$ ), $e=\{[z, a],[z, b]\},|z|=m_{i}$ and without loss of generality $z=-m_{i}, a=3, b=4$. Then we let $H_{i}$ consist of $e$ if $\{[z, 4],[z-1,4]\} \notin P_{i}$, and $e$ together with $\{[z, 4],[z-1,4]\}$ otherwise. Let $W_{i}$ be the set of edges of a path in $Z_{i}$ between the end-points of $H_{i}$ such that it contains some edges in distance at most 100 from the origin, no vertical edge of the boundary of $Z_{i}$, and as few horizontal edges of the boundary of $Z_{i}$ as possible (i.e. at most two). Let $M_{i}$ be an integer upper bound of the coupling constants of the edges incident with a vertex of $Z_{i}$. For instance $M_{1}=1$. For each edge $e$ of $Z_{i}$ such that $e \notin W_{i} \cup\left(P_{i}-H_{i}\right)$ we let $\left(J_{i+1}\right)_{e}=\left(J_{i}\right)_{e}+100 k M_{i}$, and we let $\left(J_{i+1}\right)_{e}=\left(J_{i}\right)_{e}$ otherwise.

$T_{i+1}$ is defined as folows: let $r_{i}^{\prime}$ be obtained from $r_{i}$ by deleting all the edges of $r_{i} \cap s_{i}$ which belong to $Z_{i}$. Analogously define $s_{i}^{\prime}$. Let $U_{i}^{b}\left(U_{i}^{0}\right.$ respectively) be the set of vertices of $Z_{i}-T_{i}\left(Z_{i} \cap T_{i}\right.$ respectively) such that we deleted an odd number of edges of $r_{i}$ incident with them. We let $T_{i+1}^{\prime}=\left(T_{i}-U_{i}^{o}\right) \cup U_{i}^{b}$. Observe that $T_{i+1}^{\prime}$ has no vertices in the interior of $Z_{i}$ and $r_{i}^{\prime}$ is a $T_{i+1}^{\prime}$-join and $s_{i}^{\prime}$ is a $T_{i+1}^{\prime} \cup\{x, y\}$-join. If $H_{i} \subset r_{i}^{\prime}$ or $H_{i} \subset s_{i}^{\prime}$ then let $T_{i+1}=T_{i+1}^{\prime}$ else necessarily $H_{i}$ contains two edges incident to a 'corner vertex' of $Z_{i}$ and without loss of generality assume that the vertical edge of $H_{i}$ belongs to $r_{i}^{\prime}$. In this case $T_{i+1}$ is obtained from $T_{i+1}^{\prime}$ by changing the status of both vertices of the horisontal edge of $H_{i}$. We also modify $r_{i}^{\prime}$ and $s_{i}^{\prime}$ so that we delete the horisontal edge of $H_{i}$ from $s_{i}^{\prime}$ and add it to $r_{i}^{\prime}$. Observe that $T_{i+1}$ has no vertices in the interior of $Z_{i}$ and $r_{i}^{\prime}$ is a $T_{i+1}$-join and $s_{i}^{\prime}$ is a $T_{i+1} \cup\{x, y\}$-join. Moreover $H_{i} \subset r_{i}^{\prime}$ or $H_{i} \subset s_{i}^{\prime}, r_{i}^{\prime} \Delta s_{i}^{\prime}=P_{i}$ and $r_{i}^{\prime} \cap s_{i}^{\prime}$ has no edges in $Z_{i}$. Without loss of generality assume $H_{i} \subset r_{i}^{\prime}$. Let $r_{i}^{\prime \prime}$ be obtained from $r_{i}^{\prime}$ by exchanging $H_{i}$ for $W_{i}$. Clearly $r_{i}^{\prime \prime}$ is a $T_{i+1}$-join, $r_{i}^{\prime \prime} \Delta s_{i}^{\prime}$ is a path $P_{i}^{\prime}$ obtained from $P_{i}$ by exchanging $H_{i}$ for $W_{i}$ and $E\left(s_{i}^{\prime}\right) \leq E\left(s_{i}\right)$ and $E\left(r_{i}^{\prime \prime}\right) \leq E\left(r_{i}\right)+32 M_{i} k$. Let $K_{i+1}=\left(J_{i+1}, T_{i+1}, x, y\right), r_{i+1}$ be a minimum $T_{i+1}$-join, $s_{i+1}$ be a minimum $T_{i+1} \cup\{x, y\}$ join, and let $P_{i+1}$ be the $x, y$-path in the symmetric difference of $r_{i+1}, s_{i+1}$. If $P_{i+1}$ contains an edge in distance at most 100 from the origin then let $F(K)=K_{i+1}$ otherwise we output vector $\left(K_{i+1}=\left(J_{i+1}, T_{i+1}, x, y\right), P_{i+1}, r_{i+1}, s_{i+1}, S_{i+1}, m_{i+1}, n_{i+1}, p_{i+1}\right)$. This finishes the describtion of the Procedure.

Now observe that $R=r_{i+1} \Delta\left(r_{i} \Delta r_{i}^{\prime}\right)$ is a $T_{i}$-join such that $R$ and $r_{i+1}$ differ only on the edges of $Z_{i}$. Hence $r_{i+1}$ cannot contain an edge of $Z_{i}-\left(W_{i} \cup\left(P_{i}-H_{i}\right)\right)$ since otherwise $E\left(r_{i+1}\right) \geq E(R)-32 k M_{i}+100 k M_{i} \geq E(R)+60 k M_{i} \geq E\left(r_{i}\right)+60 k M_{i}>E\left(r_{i}^{\prime \prime}\right)$. Hence $r_{i+1}$ contains all the edges of $W_{i}$ of the interior of $Z_{i}$ or none of them, and its edges from the boundary of $Z_{i}$ form a subset of $Z_{i} \cap\left[\left(P_{i}-H_{i}\right) \cup D\right]$, where $D$ is non-empty only if $H_{i}$ has a vertical 'corner' edge and then $D$ consists of one or two horizontal edges (by the definition of $W_{i}$ ). The same holds for $s_{i+1}$. If $H_{i}$ has at least one vertical edge of $Z_{i}$ then we have that $m_{i}<m_{i+1}$ or $m_{i}=m_{i+1}, n_{i}>n_{i+1}$. If $H_{i}$ has no vertical edge then it 
consists of exactly one horizontal edge and all the edges of $W_{i}$ belong to the interior of $Z_{i}$. Hence $r_{i+1}$ contains all the edges of $W_{i}$ or none of them and the edges of $r_{i+1}$ from the boundary of $Z_{i}$ belong of $P_{i}-H_{i}$. The same holds for $s_{i+1}$. Hence we have $m_{i}<m_{i+1}$ or $m_{i}=m_{i+1}, n_{i}=n_{i+1}, p_{i}>p_{i+1}$. Sumarising, $m_{i}<m_{i+1}$ or $m_{i}=m_{i+1}, n_{i}>n_{i+1}$, or $m_{i}=m_{i+1}, n_{i}=n_{i+1}, p_{i}>p_{i+1}$.

Hence after at most $(16 k)^{2}$ repetitions of the Procedure we have $F(K)$ defined. Moreover $F(K)$ is defined only if the path with desired properties exists. Finally $c_{k}>0$ exists since the set $\mathcal{Z}$ of configurations with $\left|J_{i j}\right|<1$ for $|i| \leq 4,|j| \leq k$ clearly satisfies $\mathcal{U}_{k}(\mathcal{Z}) \geq c_{k}^{\prime}>0$ and with probability one $L=F(K)$ from $\mathcal{Z}$ may be viewed as $K+\alpha_{K}$, where each component of $\alpha_{K}$ is a bounded integer and the number of possible $\alpha_{K}$ 's is bounded from above by the number of paths on vertices $[i, j],|i| \leq k,|j| \leq 4$ (which is a modest function of $k$ ). This finishes the proof.

Acknowledgement. I have started to work on it in the autumn of 2000 while I was visiting Bruno Nachtergaele in Davis: I would like to thank to him for introduction to this subject. I am indebt to Jirka Matousek for many discussions and in particular for suggesting a proof method for Pinning Lemma 2. I would also like to thank to Greg Kuperberg, Jan Vondrak, Michael Lacey, Prasad Tetali, Russell Lyons, Laszo Erdes, Daniel Stein, Charles Newman and Matteo Palassini for helpful discussions.

\section{References}

[1] S. Edwards, P.W. Anderson, J. Phys. F 5, 965 (1975).

[2] M. Mezard, G. Parisi, M.A. Virasoro, Spin Glass Theory and Beyond, World Scientific, Singapore, 1987.

[3] K. Binder, A.P. Young, Rev. Mod. Phys. 58, 801 (1986).

[4] W.L. McMillan, J. Phys. C 17, 3179 (1984).

[5] A.J. Bray, M.A. Moore, Phys. Rev. Lett. 58, 57 (1987).

[6] D.S. Fisher, D.A. Huse, Phys. Rev. Lett. 56, 1601 (1986).

[7] C.M.Newman, D.L.Stein, Nature of Ground State Incongruence in Two-Dimensional Spin Glasses, Physical Review Letters 84, 17, 3966-3969 (2000).

[8] C.M.Newman, D.L.Stein, Spatial Inhomogeneity and Thermodynamic Chaos, Physical Review Letters 76, 25, 4821-4824 (1996).

[9] M.Loebl, On Ground State Incongruence in Spin Glasses, ITI preprint series 2002094 (2002). 